\title{
Expanding therapeutic targets in bladder cancer: the PI3K/Akt/mTOR pathway
}

\author{
Christina Barbara Ching ${ }^{1}$ and Donna Elizabeth Hansel ${ }^{1,2,3,4}$
}

A complex equilibrium of biological signals exists within the human body to regulate normal cellular function and growth. Unfortunately, there are various ways in which disruption of these signaling pathways can result in uncontrollable cell growth-an important element in oncogenesis. In particular, the mammalian target of rapamycin (mTOR) pathway appears to play a central role in the development of multiple cancers, including urothelial cell carcinoma (UCC). Although often called 'a master regulator,' mTOR is but one signal in an intricate signaling cascade that controls cell growth and angiogenesis in both normal and cancerous conditions. Other important factors in this pathway include upstream activators such as phosphatidylinositol 3 kinase (PI3K) and Akt, negative regulators such as the tuberous sclerosis complex (TSC) 1/2, and downstream effectors such as p70 S6 kinase and eukaryotic initiation factor elF4E. On the basis of its important role in tumor growth, efforts have focused on developing means to effectively target the mTOR pathway in hopes of designing new treatments for various tumor types. To address the role of mTOR pathway activity in UCC, we will first review the basic elements of the PI3K/Akt/mTOR pathway and then apply this pathway to bladder cancer oncogenesis. As will be evident, significant progress has been made in defining the role of this pathway in UCC; however, continued research into the nuances of pathway regulation and the usage of targeted inhibition in bladder cancer patients is necessary to define mTOR as a promising target in this disease.

Laboratory Investigation (2010) 90, 1406-1414; doi:10.1038/labinvest.2010.133; published online 26 July 2010

KEYWORDS: mammalian target of rapamycin (mTOR); oncogenesis; phosphatase and tensin homolog (PTEN); PI3K/Akt/PTEN/mTOR pathway; urothelial cell cancer

\section{BLADDER CANCER: AN OVERVIEW}

Bladder cancer is the fifth most common cancer in the world, ${ }^{1}$ accounting for $3.2 \%$ of the worldwide cancer burden. ${ }^{2}$ Its pathogenesis involves somatic genetic changes induced by environmental carcinogens, ${ }^{3}$ such as tobacco, aromatic amines, phenacetin-containing analgesics, and arsenic. ${ }^{4,5}$ Heritable genetic factors may also contribute to oncogenesis in a subset of cases with roughly double the risk of developing cancer in first-degree relatives of patients with bladder cancer. ${ }^{4}$

In general, carcinogenesis may occur through protooncogene activation or tumor-suppressor gene loss, ${ }^{3}$ both of which have been documented in urothelial cell carcinoma (UCC), the most common form of bladder cancer. UCC is subdivided into non-invasive and invasive subtypes, with non-invasive carcinoma further subdivided into low- and high-grade lesions. ${ }^{1,3}$ Low-grade papillary urothelial cancer accounts for approximately $70-85 \%$ of UCC diagnoses ${ }^{1,6,7}$ and is characterized in most instances by either deletion of chromosome 9 or activating mutations of fibroblast growth factor receptor 3 (FGFR3), a tyrosine kinase-linked cell surface receptor important in downstream signaling of cell growth, differentiation, and angiogenesis. ${ }^{8}$ Although these lesions frequently recur, they have a low risk of progressing to invasive disease and an overall favorable prognosis. ${ }^{1,3}$ In contrast, high-grade non-invasive and invasive UCC may show loss of tumor-suppressor genes including phosphatase and tensin homolog (PTEN) and p53. ${ }^{1,3}$ Invasive UCC is often an aggressive disease with a reduced 5-year survival rate $^{1,3}$ despite treatment with radical cystectomy and adjuvant chemotherapy. ${ }^{3}$

Cell-signaling pathways have a critical role in tumorigenesis, and underlying gene or protein alterations in these pathways can influence cell cycle control, DNA repair, and carcinogen metabolism. ${ }^{4}$ In particular, a subset of mammalian target of rapamycin (mTOR) pathway alterations have

\footnotetext{
${ }^{1}$ Glickman Urological and Kidney Institute, Cleveland Clinic, Cleveland, OH, USA; ${ }^{2}$ Pathology and Laboratory Medicine Institute, Cleveland Clinic, Cleveland, OH, USA; ${ }^{3}$ Taussig Cancer Institute, Cleveland Clinic, Cleveland, OH, USA and ${ }^{4}$ Genomic Medicine Institute, Cleveland Clinic, Cleveland, OH, USA Correspondence: Dr DE Hansel, MD, PhD, Pathology and Laboratory Medicine Institute, 9500 Euclid Avenue, Desk L25, Cleveland Clinic, Cleveland, OH 44195, USA. E-mail: hanseld@ccf.org 
been shown to occur in bladder cancer ${ }^{9}$ and appear to influence tumor behavior. As a result, the potential for further exploration of this pathway in bladder cancer is of interest given the potential for prognostic information and targeted therapy.

\section{mTOR AND ITS ROLE IN CELLULAR PROCESSES}

mTOR is a $239 \mathrm{kDa}$, ubiquitously expressed, evolutionarily conserved, serine/threonine protein kinase. ${ }^{10,11}$ It was originally identified as the target of the antibiotic rapamycin (Rapamune, Wyeth pharmaceuticals, Madison NJ, USA). ${ }^{12}$ Subsequent studies revealed a broad spectrum of functions for this molecule, including cell cycle progression, cell proliferation, angiogenesis, and apoptosis. ${ }^{13-15}$ mTOR also plays an instrumental role in protein translation initiation, the rate-limiting step of protein synthesis, by enabling the recruitment of ribosomes to mRNA by eukaryotic initiation factor (eIF). ${ }^{13}$ As such, mTOR activates its downstream mediator ribosomal S6 kinase (p70 S6 kinase-1) and is responsible for the progression of the cell from G0/G1 to $S$ phase. ${ }^{11,13}$

The mTOR pathway also has an important role in energy balance and coordinates cell growth based on the nutritional and energy status of the cell. mTOR uses adenosine triphosphate (ATP), adenosine monophosphate, and inorganic polyphosphate levels to monitor the cell's energy status for protein synthesis. ${ }^{16,17}$ It also integrates growth stimuli with cell cycle progression, initiating mRNA translation and thus cell proliferation only in a favorable environment with adequate supporting nutrients. ${ }^{12,16}$ In the presence of abundant growth factors and nutrients, there is an increase in phosphorylation and activation of molecules crucial to protein translation. ${ }^{18,19}$ Conversely, phosphorylation can be decreased during times of environmental stress, nutrient deficiency, and even in the presence of viruses. ${ }^{13}$

mTOR exists in two different complexes: mTORC1 (mTOR complex 1) and mTORC2 (mTOR complex 2). mTORC1 is a heterotrimeric protein kinase composed of mTOR kinase, regulatory associated protein of mTOR (raptor), and mLST8 (also known as $\mathrm{G} \beta \mathrm{L}$ ), and is distinguished by its sensitivity to rapamycin. Raptor was the first protein shown to bind to mTOR and is a scaffolding protein required for downstream-signaling activity. It allows for phosphorylation of such proteins as p70 S6 kinase (p70 S6K) and initiation-binding protein $4 \mathrm{E}-\mathrm{BP} 1 .^{16}$ By phosphorylating $4 \mathrm{E}-\mathrm{BP} 1, \mathrm{mTOR}$ releases eIF4E, an important initiation factor, to participate in the assembly of the translational initiation complex. mTORC2 consists of four subunits that include mTOR kinase, mLST8, rapamycin-insensitive companion of mTOR (rictor), and mSin-1 (mitogen-activated protein kinase (MAPK)-associated protein 1). Unlike mTORC1, mTORC2 is rapamycin insensitive. ${ }^{14,20}$ It regulates actin cytoskeleton remodeling, whereas mTORC1 is primarily responsible for nutrient and growth factor signal sensing. ${ }^{14,20-22}$

\section{MTOR PATHWAY ACTIVATION}

mTOR is only one part of the larger phosphatidylinositol 3 kinase (PI3K)/Akt/mTOR pathway (Figure 1). Upstream activation of this pathway begins when growth factors (insulin, insulin-like growth factor, platelet-derived growth factor), mitogens, hormones, or nutrients activate PI3K. ${ }^{20,22,23} \mathrm{PI} 3 \mathrm{~K}$ is a heterodimer that consists of a catalytic subunit ( $\mathrm{p} 110)$ and a regulatory subunit (p85, p55, or p50). ${ }^{22}$ The activated p110 catalytic subunit, encoded by the PIK3CA gene, phosphorylates phosphatidylinositol bisphosphate ${ }^{4,5}$ on the inner leaflet of the cytoplasmic membrane to generate phosphatidylinositol triphosphate (PIP3). ${ }^{3-5}$ PIP3 subsequently binds to the pleckstrin homology domain of Akt1 kinase (also known as protein kinase B) to recruit Akt1 to the cell membrane for phosphorylation. ${ }^{19,24}$ Aktl is phosphorylated at Threonine 308 by phosphoinositide-dependent protein kinase $1(\mathrm{PDK}-1)^{25-27}$ and at Serine 473 (Ser473) by PDK-2, which is believed to be rictor. ${ }^{9,14,19,27}$ Activated Akt1 inactivates the tuberous sclerosis complex (TSC) composed of TSC1 and TSC2, by directly phosphorylating the GTPase-activating protein TSC2. TSC2 and TSC1 normally act together as tumor-suppressor proteins inhibiting cell growth by negatively regulating the small GTPase-activating protein Ras homolog enriched in brain (Rheb). ${ }^{14}$ When released from TSC inhibition, Rheb is free to activate mTORC1. ${ }^{19,22,28}$

Activated mTOR can subsequently phosphorylate p70 S6 kinase-1 (p70 S6K) and 4E-BP. p70 S6K, a serine/threonine kinase, phosphorylates the ribosomal protein $\mathrm{S} 6$ of the $40 \mathrm{~S}$ ribosomal subunit at Ser235 and 236 to increase translation of $5^{\prime}$ terminal oligopyrimidine tract mRNAs ${ }^{10}$ and also binds to mitochondrial membranes and inactivates the pro-apoptotic molecule BAD. ${ }^{11,20}$ Phosphorylation of 4E-BP allows assembly of the translational initiation complex with subsequent translation of proteins necessary for progression of the cell cycle past the G1 phase, such as cyclin D1. ${ }^{23,27,28}$

\section{NEGATIVE REGULATION OF THE mTOR-SIGNALING PATHWAY}

PTEN is a tumor suppressor and lipid/protein phosphatase, mapped to chromosome 10 , that is commonly mutated or lost in solid tumor malignancies. ${ }^{21}$ Furthermore, PTEN has differential functions based on its location in either the nucleus or cytoplasm. ${ }^{29}$ In the cytoplasm, PTEN uses its lipid phosphatase activity to regulate cell-signaling processes through the PI3K/Akt/mTOR pathway, ${ }^{21}$ having a role in apoptosis and cell growth. Specifically, PTEN dephosphorylates PIP $3,{ }^{25,30}$ preventing Akt1 activation. In contrast, nuclear PTEN has been found to regulate cell cycle progression and maintain chromosomal stability independently of Akt regulation. ${ }^{29,31}$ Not only has PTEN been found to localize to the nucleus during various phases of the cell cycle, resulting in higher levels during the G0-G1 phase vs lower levels in S phase, ${ }^{32}$ but it actually downregulates cyclin D1 expression and MAPK phosphorylation, resulting in cell cycle 


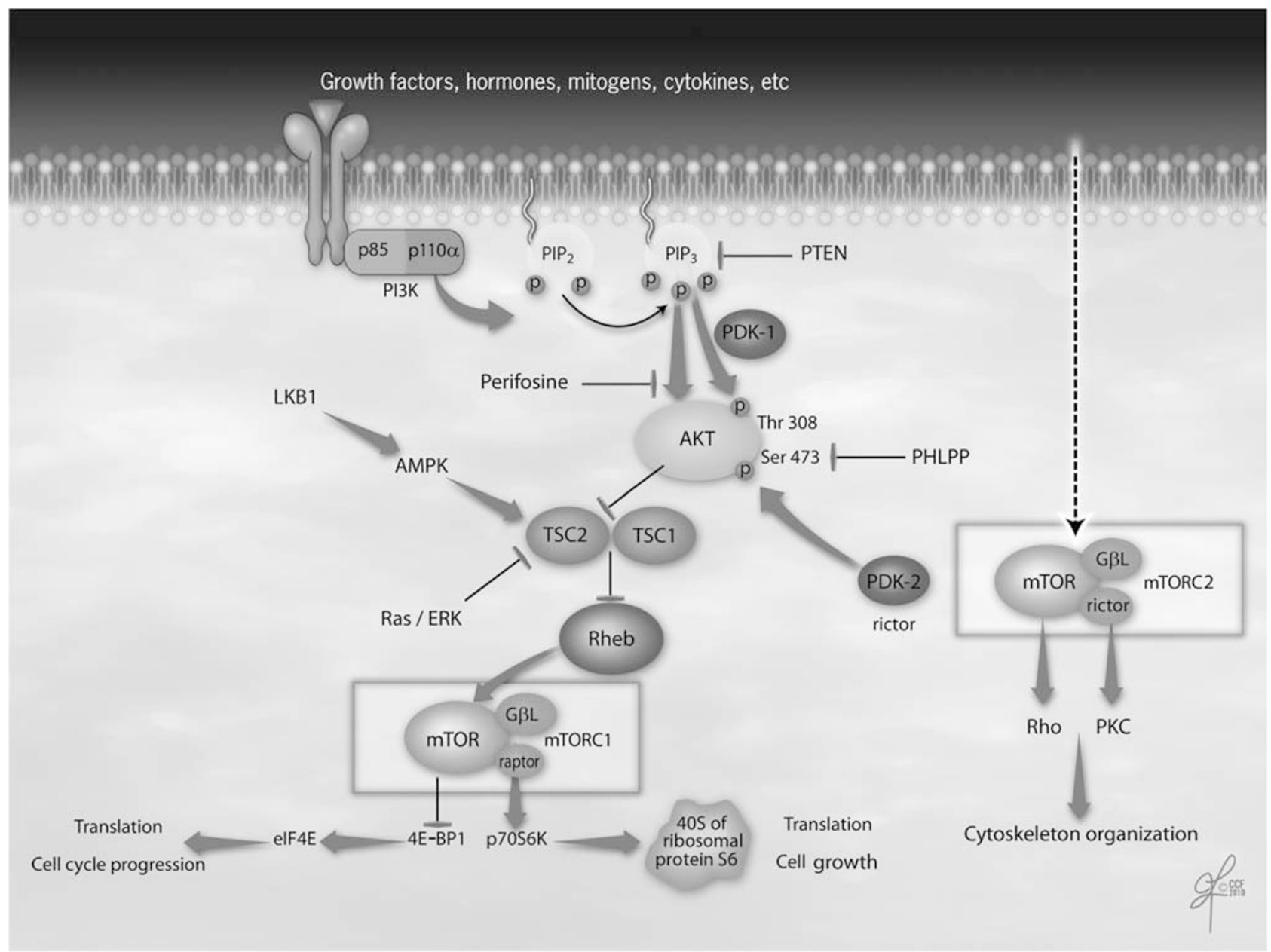

Figure 1 Schematic of the PI3K/Akt/mTOR pathway. Reprinted with permission, Cleveland Clinic Center for Medical Art \& Photography (C) 2009. All rights reserved.

arrest. ${ }^{33}$ Nuclear PTEN has also been found to localize to the centromere and specifically associate with an integral component of the kinetochore (CENP-C), maintaining centromere stability and preventing breakage. PTEN loss results in up to a sevenfold increase in spontaneous DNA double-strand breaks as compared with intact PTEN. ${ }^{31}$ These differences in subcellular localization of PTEN are thought to be an important factor in neoplastic progression, given that cytoplasmic or nuclear expression varies between normal and oncologic tissue. ${ }^{29}$

Negative feedback loops also play a critical role in mTOR pathway regulation and may in part be responsible for observed mTOR inhibition or rapamycin 'resistance.' For example, studies have found that treatment with the mTOR inhibitor rapamycin actually results in increased Akt activation and eIF4E phosphorylation. ${ }^{34}$ mTORC1 and p70 S6K are part of a negative feedback loop with PI3K/Akt, ${ }^{19,22}$ controlling the response of $\mathrm{PI} 3 \mathrm{~K}$ to insulin through insulin receptor substrate (IRS) proteins. IRS-1 is normally phosphorylated and activated by p70 S6K. This same phosphorylation, however, also promotes IRS-1 degradation and expression reduction, decreasing insulin-based activation of $\mathrm{PI} 3 \mathrm{~K}$. As a result, $\mathrm{PI} 3 \mathrm{~K}$ activation and signal duration is thus regulated. ${ }^{22}$ mTORC1 inhibition can interrupt this feedback loop, resulting ultimately in feedback activation or upregulation of PI3K/Akt activity. ${ }^{19,35}$ This has been seen in cases of chronic insulin exposure in which the normal decrease in IRS-1 protein is prevented with rapamycin treatment, ${ }^{22}$ thus resensitizing the PI3K/Akt/mTOR pathway to insulin. ${ }^{36}$

This complex interaction between mTORC1 and PI3K/Akt may account for some of the ineffectiveness of mTOR inhibition seen in cancer therapy ${ }^{37}$ and thus support the concept of multi-targeted therapy. Various studies have looked at mTOR inhibition in combination with direct Akt (ie perifosine) or PI3K inhibition (ie LY294002), or even through other signaling pathways such as the MAPK/extracellular signal-regulated kinase pathway or epidermal growth factor receptor inhibition. ${ }^{34,38-40}$ Studies have found that such combinations result in synergistic effects on cell culture growth, ${ }^{34}$ while also giving concern for possible increased benign tissue toxicity because of the pan-interruption of these critical signaling networks. 
Recent interest has focused on the role of the mTORC2 complex and its potential role in regulating the PI3K/Akt/ mTOR pathway. We know that mTORC2 is responsible for the direct phosphorylation activation of Akt at Ser473. ${ }^{19}$ As a result, mTORC2 could be an important indirect regulator of $\mathrm{mTORC} 1^{14}$ with potentially more specific targeting capabilities, given that mTORC2 activity appears specific to cancer cells with a less essential role in normal cells. ${ }^{41}$ Ideally, this would lessen the risk of side effects and provide a more favorable therapeutic window. In addition, mTORC2-specific inhibition would not interrupt the negative feedback loop seen with mTORC1 and thus improve efficacy. Although mTORC2-specific inhibitors do not yet exist, they appear to be an exciting and potentially beneficial next generation of targeted therapy.

\section{ONCOGENESIS AND THE mTOR PATHWAY}

As reflected in earlier sections, the mTOR pathway has a significant role in cell growth and proliferation. Dysregulation of any step along the pathway can thus result in altered cell dynamics and ultimately tumor development. ${ }^{17,21}$ Alterations in the mTOR pathway are common in numerous malignancies, including prostate cancer, melanoma, malignant glioma, endometrial cancer, renal cell cancer, breast cancer, medulloblastoma, alveolar rhabdomyosarcoma, and mantle cell lymphoma. ${ }^{10}$ In general, increased activity of this pathway-either by loss of inhibitory steps or increased activity of growth-promoting elements-can potentially promote tumorigenesis.

Chen et al performed one of the first studies to look at the role of germline genetic variations in the PI3K pathway in bladder cancer development. They evaluated 231 single-nucleotide polymorphisms (SNPs) in 19 genes in PI3K/Akt/ mTOR-signaling looking for predictors of bladder cancer risk. They found four SNPs in raptor that were significantly associated with developing bladder cancer (rs11653499, OR: 1.79; rs7211818, OR: 2.13; rs7212142, OR: 1.57; rs9674559, OR: 2.05) and that an increasing number of unfavorable genotypes further increased the risk of developing cancer. When evaluated for potential interactions between genetic variants and patient demographics, they found a significant interaction in older, male subjects who had never or were light smokers. Although they did not find that heavy tobacco use was associated with these SNPs, they hypothesized that heavy smoking is a strong enough environmental risk factor that it might overpower what contribution genetic variation has in the risk of developing bladder cancer. ${ }^{4}$

\section{PTEN AS A TUMOR SUPPRESSOR IN BLADDER CANCER}

PTEN is a potent inhibitor of the PI3K/Akt/mTOR pathway; PTEN alterations have been described in glioblastoma, melanoma, endometrial cancer, breast cancer, prostate cancer, and bladder cancer. ${ }^{13,17,28,42}$ PTEN also functions to regulate chemotaxis and cell motility, mechanisms that promote tumor invasion. ${ }^{9,43-45}$ As a result, it is not surprising that
PTEN loss is associated with aggressive tumor growth, metastases, and worsened patient outcomes. ${ }^{46,47}$ In invasive UCC, PTEN is mutated or shows loss of heterozygosity (LOH) in up to $30 \%$ of cases, ${ }^{9,48,49}$ whereas this number falls to $6.6 \%$ in superficial tumors $(\mathrm{pTa} / \mathrm{pT} 1) .{ }^{42}$ One group actually reported reduced PTEN protein expression in $94 \%$ of advanced stage bladder cancer specimens, whereas only $42 \%$ of superficial tumors and $8 \%$ of carcinoma in situ (CIS) specimens had reduced PTEN expression. ${ }^{30}$

Researchers have also found that PTEN status may be important in influencing response to treatment. One study found a reduced sensitivity to the PI3K inhibitor LY294002 in prostate and bladder cancer cell lines with intact PTEN expression as compared with those cells that lacked functional PTEN. ${ }^{46}$ As a result, PTEN status appears to mediate resistance to PI3K pathway-directed therapy; conversely, the loss of PTEN expression may actually represent a useful background to maximize effects of PI3K inhibition.

In addition to its direct effects on cancer cell processes, PTEN may also have downstream effects via interaction with the p53 pathway, which is commonly mutated in bladder cancer. ${ }^{1}$ Specifically, combined PTEN and p53 inactivation may promote bladder tumorigenesis, at least in a subset of cases. Using a mouse model that harbored loss of both p53 and PTEN, mice frequently developed invasive UCC, with metastases to local lymph nodes and distant sites. In contrast, p53 or PTEN loss alone did not lead to tumor formation. The authors concluded that combined p53 and PTEN loss likely increased mTOR signaling and showed a dramatic reduction in tumor growth with mTOR inhibition. ${ }^{1}$ Concurrent analysis of patient specimens identified primary p53 alterations and PTEN downregulation in invasive UCC, with $41 \%$ of samples showing concurrent alterations. This combined loss of p53 and PTEN was associated with an especially poor patient outcome, with a median survival of 5 months $v s 6$ years in those with p53 mutation alone $(p<0.001)$.

\section{PI3K MUTATIONS CHARACTERIZE A SUBSET OF BLADDER CANCERS}

Although the PI3K/Akt pathway can be dysregulated by loss of PTEN as discussed above, mutations in PI3K itself may result in its constitutive activity. One of the most common mutations specific to PI3K is in PIK3CA, which encodes the catalytic p110 $\alpha$ subunit of PI3K. Mutations in it are almost exclusively gain-of-function ${ }^{50}$ and have been identified in about $12 \%$ of all human cancers. ${ }^{51}$ They have specifically been found in breast, cervical, ovarian, gastric, colorectal, and lung, ${ }^{20}$ and in up to $27 \%$ of UCCs. ${ }^{49}$ A study evaluating renal and collecting system diseases found that PIK3CA mutations were specific to UCC of the renal pelvis as compared with other renal neoplasms such as renal cell carcinoma. These mutations were present in $13.6 \%$ of patients with upper tract UCC and absent in patients with other pathologies. ${ }^{52}$ 
The presence of a PIK3CA mutation, however, appears correlated with low-grade and low-stage urothelial disease. ${ }^{53}$ The mutations are strongly associated with FGFR3 mutations found prevalent in superficial bladder tumors, showing that both mutations may be involved in the same pathway of tumor progression..$^{53}$ As a result, mutations in PIK3CA may prove to be of prognostic use in identifying the clinical biology of the disease. ${ }^{53}$ On the other hand, the possibility of multiple mutations in the PI3K pathway-ie concurrent PTEN, TSC1, loss of 9q heterozygosity, etc-makes detailed analysis of this pathway challenging and may result in using multi-agent or multimodal therapy for this disease. ${ }^{49}$

For the most part, mutations in PIK3CA are restricted to certain 'hot spots' in the gene-specifically in the helical and kinase domains. For example, in cancers such as breast, endometrial, prostate, and colon, $80 \%$ of mutations occur in one of three such 'hot spots' of which two are in the helical and one is in the kinase domain. ${ }^{54}$ The distribution of mutations is a little different in UCC; nine specific mutations in PIK3CA have been identified in UCC, with the most common mutations being E542K (24\%) and E545K (52\%) of the helical domain. In contrast, mutation in the kinase domain H1047R, which in other cancers is the most common (46\%), is not as common in UCC (only 13\%). ${ }^{48,49}$

Although the majority of mutations of PIK3CA are in the catalytic domain, mutations have been observed in the p $85 \alpha$ regulatory subunit (PIK3RI) of PI3K. Normally, it is the interaction between the two subunits of PI3K, p110 and p85, that is important in regulating PI3K activity; phosphorylation of $\mathrm{p} 85 \alpha$ regulates PI3K activity through suppression of $\mathrm{p} 110$. One can see, then, how mutations in $\mathrm{p} 85 \alpha$ could thus disrupt this interaction and result in increased enzyme activity. ${ }^{55}$ In a study performed by Philp et al ${ }^{56}$ mutations specifically in the phosphorylation site (Ser608) on $\mathrm{p} 85 \alpha$ were partially responsible for elevated phosphorylated-Akt (P-Akt) levels (a marker of PI3K activity) in two ovarian and two colon cancer cell lines. Although mutations in $\mathrm{p} 85 \alpha$ have been identified in primary human glioblastoma, ovarian cancer, and colon cancer, ${ }^{56,57}$ they have not yet been studied in UCC.

\section{Akt MUTATIONS AND PHOSPHORYLATION}

Akt exists as three different isoforms: Akt-1, 2, and 3. Although structurally homologous, they differ by their sites of phosphorylation required for their activation. ${ }^{58}$ Akt activity appears significantly increased in UCC, although for the most part, series consist of small sample sizes. One study of only 20 primary UCC samples found a significantly higher presence of P-Akt in 55\% of UCC tumor specimens as compared with normal urothelium. ${ }^{59}$ Another study evaluated gene expression profiling of 13 upper tract UCC and found that $76.9 \%$ had an Akt activation-specific expression signature. ${ }^{52}$ A larger study of 251 UCC tissue microarray samples found that P-Akt was selectively expressed in bladder cancer as compared with normal urothelium. It was 'highly expressed'-defined as intermediate or strong staining- however, in only $5-6 \%$ of UCC samples. ${ }^{60}$ Although Akt and P-Akt levels are not significantly associated with tumor grade or stage, ${ }^{60}$ increased activity likely affects downstream mTOR activation and output.

Additional proposed roles for increased Akt activity include inhibition of apoptosis and alterations in methylation status. Elevated levels of active Akt have been proposed to mediate resistance to the pro-apoptotic cytokine tumor necrosis factor-related apoptosis-inducing ligand (TRAIL). ${ }^{61}$ Under normal conditions, TRAIL activity results in caspase activation, mitochondrial depolarization, and apoptosis. A study by Oka et $a l^{61}$ found that elevated levels of Akt protected bladder cancer cell lines from TRAIL-induced apoptosis and was reversible upon PI3K inhibition. Furthermore, Akt activation limited mitochondrial cytochrome-c release and caspase-mediated apoptosis, which imbued resistance to paclitaxel in the UCC T24 cell line. Conversely, Akt inhibition helped sensitize these cells to chemotherapy treatment. ${ }^{62}$

Akt may also serve a role in DNA methylation, which is catalyzed by DNA methyltransferase 1 (DNMT1) and affects gene expression and chromatin stability. DNMT1 is the principle enzyme to maintain $\mathrm{CpG}$ (cytosine followed by guanine) methylation and is required for somatic cell survival and development. In cancer, tumor-suppressor genes are often aberrantly hypermethylated because of increased levels of DNMT1. When investigating the relationship between DNA methylation and Akt activity, a positive correlation was identified between Ser473 phosphorylation and DNMT1 protein levels in both primary bladder cancer specimens and bladder cancer cell lines. Akt likely increased DNMT1 levels through inhibition of the proteasome pathway that is normally responsible for DNMT1 degradation. During Akt inhibition, DNMT1 levels decreased, resulting in DNA hypomethylation. As a result, this study concluded that Akt activity was positively correlated with DNMT1 expression, enabling, and maintaining DNA methylation and chromatin stability, thus contributing to cell growth. ${ }^{63}$

Finally, increased activity of Akt may also be attributable to gene mutations and such mutations have been previously reported in breast, colorectal, ovarian, and lung cancer. Mutations in Akt1 have been best evaluated in bladder cancer and generally consist of single point mutations. ${ }^{64}$ The G49A (E17K) mutation occurs in $4.8 \%(2 / 42)$ of bladder cancer cell lines and $2.7 \%(5 / 184)$ of bladder tumors and appears to increase Akt1 protein membrane localization and activation. A second mutation at G145A (E49K) was also identified in one of the bladder cancer cell lines (2.4\% incidence) and may result in Akt1 activation and may possibly synergize with the G49A mutation. ${ }^{64}$ However, given the rarity of these mutations, they are unlikely to significantly contribute to the development of UCC in the majority of patients. ${ }^{48,64}$

\section{TSC1 MUTATIONS}

The TSC complex (TSC1/TSC2) acts directly downstream of Akt and is important in mTOR inhibition. Mutations or loss 
of either TSC1, on chromosome 9q34, or TSC2, on chromosome 16p13.3, result in the tuberous sclerosis syndrome. ${ }^{17,18,28}$ In bladder cancer, chromosome 9 is frequently affected by deletions; ${ }^{3} \mathrm{LOH}$ of chromosome 9 occurs in $>50 \%$ of bladder tumors, regardless of grade and stage ${ }^{65}$ with loss at 9q34 especially common. ${ }^{66}$ Not surprisingly, loss of TSC1 function occurs in $14.5 \%$ of UCCs, ${ }^{48,66}$ resulting in increased mTOR signaling and resistance to apoptosis. ${ }^{3}$ The TSC2 portion of this inhibitory complex also has a critical role in mTOR inhibition and germline TSC2 are approximately five times more common in sporadic forms of the tuberous sclerosis syndrome. ${ }^{67}$ Unfortunately, mutations in TSC2 have not been characterized in detail in UCC.

\section{ROLE OF MTOR IN BLADDER CANCER}

Increased mTOR signaling has been shown to promote more aggressive disease behavior in various cancers such as cervical and gastric cancer, ${ }^{68-70}$ and the effect of mTOR on cellular function have recently been evaluated in UCC. ${ }^{71}$ One recent study has identified phospho-S6-a marker of mTOR activity-expression in 55\% of muscle-invasive UCCs, with increased expression evident in paired lymph node metastases. The mTOR pathway activity was associated with reduced patient survival and increased pathological stage. Inhibition of mTOR signaling with rapamycin reduced cell proliferation and migration in vitro and reduced tumor volume in a T24-xenograft model by $55 \% .^{71}$ These results were supported by a second study that showed mTOR activation in muscle-invasive UCC, ${ }^{1,72}$ with $\mathrm{mTOR}$ inhibition preventing the progression of CIS to invasive bladder cancer. ${ }^{72}$

Another important role of mTOR in tumor biology is the induction of angiogenesis. mTOR regulates the expression of hypoxia inducible factor and thus subsequently of vascular endothelial growth factor (VEGF) ${ }^{20}$ VEGF is a well-characterized vascular growth factor and is secreted at high levels in TSC1 and TSC2 null mice, supporting the upstream role of mTOR in VEGF production. These results support the antiangiogenic effects documented with mTOR inhibitors, such as rapamycin, in patients undergoing treatment with mTOR inhibition in renal cell carcinoma. ${ }^{17}$

\section{TARGETING THE MTOR PATHWAY}

The mTOR pathway is an attractive target for clinical treatment of numerous cancers, given its central role in cell proliferation, apoptosis, and angiogenesis. ${ }^{12,28}$ Inhibition of mTOR itself has been a heavily investigated area in tumor treatment and numerous compounds are available that target this molecule.

Rapamycin (Rapamune) was discovered in the 1970s as a natural macrolide lactone antifungal agent purified from Streptomyces hygroscopicus. ${ }^{11-13,17,18}$ It was initially used as an immunosuppressant, ${ }^{13}$ though was later found to have potent antitumor activity. ${ }^{12}$ Effects of rapamycin include its ability to inhibit angiogenesis, as well as reducing cell proliferation. ${ }^{11,16}$ Rapamycin complexes with the $12-\mathrm{kDa}$ intracellular FK506-binding protein (FKBP12) before binding to the FKBP12-rapamycin-binding domain of mTOR. This domain appears to flank the mTOR-kinase domain, thus inhibiting p70 S6K and 4E-BP1 phosphorylation and resulting in G1 arrest. ${ }^{12,13,17}$ Although the mechanism by which rapamycin actually inhibits mTOR-kinase activity is somewhat unclear, ${ }^{13}$ it may function by destabilizing the mTOR-raptor complex, interfering with mTOR regulatory proteins ${ }^{17}$ or activating phosphatases against downstream molecules. ${ }^{13}$ Rapamycin primarily inhibits mTORC1, although prolonged exposure has been shown to disrupt both mTORC2 and Akt signaling. ${ }^{73}$

Several mTOR inhibitor derivatives (rapalogs) have been developed: temsirolimus or CCI-779 (Tonsel, Wyeth pharmaceuticals, Madison NJ, USA), everolimus or RAD 001 (Afinitor, Novartis Pharma, Basel, Switzerland), and deforolimus or A23573 (Ariad Pharma, Cambridge, MA, USA). ${ }^{74}$ These compounds use the same inhibitory mechanism as rapamycin and are considered as potent in their antitumor capabilities. The primary difference between these compounds is their bioavailability: ${ }^{11,23}$ temsirolimus and deforolimus are water soluble and can thus be given intravenously, whereas rapamycin and everolimus have low solubility and must be given orally. The rapalogs are primarily metabolized in the liver by the CYP450 mechanism. ${ }^{23}$ The major doselimiting toxicity is mucositis, although skin toxicities and myelosuppression are also important factors. ${ }^{11}$

Effects of mTOR inhibition on tumor growth in patients have been most successfully demonstrated in metastatic renal cell carcinoma, with temsirolimus recently FDA approved for the treatment of this disease. ${ }^{9,75,76}$ Although clinical trials evaluating mTOR inhibition in UCC have been slow to develop, the need for new therapies for bladder cancer make this an interesting potential target for future studies. ${ }^{9}$

\section{FUTURE DIRECTIONS-OTHER THERAPIES}

The last 5 years have seen an explosion of interest in mTOR. Success in its targeted inhibition, as seen in metastatic renal cell carcinoma, ${ }^{75,76}$ has raised the possibility of mTOR inhibition in the treatment of other cancers. In addition to rapamycin analogs, other mechanisms by which to target mTOR have also been described in the literature. For example, farnesylation of Rheb through farnesyltransferase is necessary to promote mTOR signaling, and the farnesyltransferase inhibitor L-744832 (Enzo Life Sciences International, Plymouth Meeting, PA, USA) has been shown to prevent Rheb activity and thus p70 S6K activation. ${ }^{17}$ Another example is (-)-epigallocatechin gallate, a phytochemical in green tea, that has been seen to reduce T24 UCC cell proliferation and viability in a dose- and time-dependent manner thought because of inactivation of the PI3K/Akt pathway. ${ }^{77,78}$

Perhaps, some of the more popular alternatives to mTORspecific inhibition have been in targeting PI3K and Akt. Although clinical studies evaluating the role of PI3K inhibition 
in bladder cancer are lacking, PI3K inhibitors have contributed extensively to our understanding of the PI3K/Akt pathway and are currently under clinical investigation for other cancers.

PI3K inhibition is comprised of both pan-PI3K inhibitors and isoform-specific inhibitors. ${ }^{79}$ Perhaps the most well known are the first generation of PI3K inhibitors, LY294002 (Enzo Life Sciences, Farmingdale, NY, USA) and Wortmannin (Fermentek, Jerusalem, Israel), both of which were first described in the early 1990s. ${ }^{80}$ Although neither have been clinically relevant because of poor solubility and a poor side effect profile, ${ }^{25,80}$ they have been instrumental in contributing to our understanding of the PI3K-signaling pathway and its role in bladder cancer pathology. ${ }^{81}$ Wortmannin and LY294002 both act through the PI3K ATP-binding site; Wortmannin is a non-competitive irreversible inhibitor, whereas LY294002 is a competitive PI3K inhibitor. ${ }^{25,80}$ LY294002 is thought to be more specific and more stable than Wortmannin and has thus been used more often to define PI3K signaling. ${ }^{80,81}$ LY294002 has shown successful in vitro dose-dependent cell growth inhibition in ovarian cancer $^{82}$ and reduced invasion capacity in bladder cancer cell lines. ${ }^{59}$ Acutely, it has shown inhibition of Akt1 phosphorylation in bladder cancer cell lines; however, response with chronic exposure depended on PTEN status. ${ }^{46}$ Unfortunately, LY294002's dermal toxicity and risk of severe respiratory depression limits its use in the clinical setting. ${ }^{82,83}$

There are currently other pan-PI3K inhibitors in evaluation; however, none specifically examine effects in bladder cancer. These inhibitors include PX-866 (Compound 14, Oncothyreon, Seattle, WA, USA), a Wortmannin analog being evaluated in human ovarian cancer, lung cancer, and intracranial glioblastoma; SF1126 (Compound 15, Semafore Pharmaceuticals, Westfield, IN, USA), an LY294002 prodrug with promise in glioma and prostate cancer; and NVPBEZ235 (Compound 18, Novartis, Switzerland) with possible use in breast, prostate, and brain cancers. Most of these are in phase I studies and require further research to determine efficacy and safety. ${ }^{84}$

Given that pan-PI3K inhibitors are commonly associated with immunosuppression and glucose intolerance, ${ }^{48}$ isoformspecific inhibitors, targeting specific catalytic subunits, ${ }^{80}$ have been investigated in the hopes of having less side effects, while maintaining efficacy in targeted inhibition. ${ }^{79}$ They may be successful in targeting PIK3CA mutations if $\mathrm{p} 110 \alpha$ specific. $^{79}$ There are very few PI3K $\alpha$ inhibitors with some of the most selective PI3K $\beta$ inhibitors including TTGX-126, TGX-221 (Cayman Chemical, Ann Arbor, MI, USA), and TGX-286. ${ }^{80}$

One of the main interests in developing PI3K inhibitors is the possibility of using them in combined or dual therapy (ie PI3K and mTOR inhibitors), once again trying to preserve efficacy while reducing potential side effects. Given that dysregulation can occur at multiple points in the PI3K/Akt/ mTOR pathway and any single point of inhibition could actually result in increased activity in other areas of the pathway because of the complicated feedback system briefly discussed before, dual therapy is likely going to be more effective than monotherapy. Dual PI3K-mTOR inhibitors are the most common so far, given that the p110 subunit of PI3K and mTOR share similar structures and thus inhibitors of one likely inhibit the other. ${ }^{48,79}$ Some dual PI3K-mTOR inhibitors include PI-103 (Echelon Biosciences, Salt Lake City, UT, USA), ${ }^{84}$ BEZ235 (Novartis Oncology, East Hanover, NJ, USA), and BGT226 (Novartis Oncology). ${ }^{85}$ All are early in development without specifically having looked at their use in bladder cancer.

PI3K inhibitors may not control Akt activation in those with inherent Akt mutations or amplification. As a result, specific Akt inhibitors have been developed. Akt inhibitors include non-catalytic site inhibitors and ATP mimetics. ${ }^{79}$ The Akt inhibitor perifosine (KRX-0401, Keryx Biopharmaceuticals, New York, NY, USA) has been investigated in one phase I trial that included a patient with bladder cancer. ${ }^{86}$ Perifosine is a lipid-based phosphatidylinositol analog that prevents binding of Akt to PIP3 ${ }^{85}$ This study specifically evaluated perifosine used in combined treatment with radiation in solid tumor patients and found that perifosine could be used safely, but it is awaiting a phase II evaluation. ${ }^{86}$

\section{CONCLUSION}

The mTOR pathway has important roles in controlling cell metabolism, survival, proliferation, and angiogenesis under normal and cancerous conditions. Numerous factors within the mTOR-signaling cascade appear to be altered in bladder cancer and may serve as potential predictive or therapyrelated markers in this disease. Although most of the work on targeted therapy in the mTOR pathway has specifically focused on mTOR inhibition, new pharmaceutical agents that target upstream factors are becoming increasingly available and may serve a role in single agent or multimodal therapy for bladder cancer. Ongoing research is important to further delineate the role of this pathway in bladder cancer to not only understand the underlying disease biology, but also to streamline new agents that can be used for therapeutic purposes.

\section{ACKNOWLEDGEMENTS}

This work was funded by a pilot grant from the American Cancer Society, Ohio Division (DEH), and a KL2 Career Development Award (DEH). It was also supported by the Cleveland Clinic Research Programs Committee Grant Award \#2009-1034 (CBC).

\section{DISCLOSURE/CONFLICT OF INTEREST}

The authors declare no conflict of interest.

1. Puzio-Kuter AM, Castillo-Martin M, Kinkade CW, et al. Inactivation of p53 and PTEN promotes invasive bladder cancer. Genes Dev 2009; 23:675-680.

2. Mitra AP, Datar RH, Cote RJ. Molecular pathways in invasive bladder cancer: new insights into mechanisms, progression, and target identification. J Clin Oncol 2006;24:5552-5564. 
3. Abraham R, Pagano F, Gomella LG, et al. Chromosomal deletions in bladder cancer: shutting down the pathways. Front Biosci 2007; 12:826-838.

4. Chen M, Cassidy A, Gu J, et al. Genetic variations in PI3K-AKT-mTOR pathway and bladder cancer risk. Carcinogenesis 2009;30:2047-2052.

5. Negri E, La Vecchia C. Epidemiology and prevention of bladder cancer. Eur J Cancer Prev 2001;10:7-14.

6. Knowles MA. What we could do now: molecular pathology of bladder cancer. Mol Pathol 2001;54:214-221.

7. Skinner DG, Stein JP, Lieskovsky G, et al. 25-year experience in the management of invasive bladder cancer by radical cystectomy. Eur Urol 1998;33:25-26.

8. Kanai M, Goke M, Tsunekawa S, et al. Signal transduction pathway of human fibroblast growth factor receptor 3. J Biol Chem 1997; 272:6621-6628.

9. Garcia JA, Danielpour D. Mammalian target of rapamycin inhibition as a therapeutic strategy in the management of urologic malignancies. Mol Cancer Ther 2008;7:1347-1354.

10. Iwenofu OH, Lackman RD, Staddon AP, et al. Phospho-S6 ribosomal protein: a potential new predictive sarcoma marker for targeted mTOR therapy. Mod Pathol 2008;21:231-237.

11. Vignot $S$, Faivre $S$, Aguirre $D$, et al. mTOR-targeted therapy of cancer with rapamycin derivatives. Ann Oncol 2005;16:525-537.

12. Panwalkar A, Verstovsek S, Giles FJ. Mammalian target of rapamycin inhibition as therapy for hematologic malignancies. Cancer 2004; 100:657-666.

13. Gingras A, Raught B, Sonenberg N. Regulation of translation initiating by FRAP/mTOR. Genes Dev 2001;15:807-826.

14. Guertin DA, Sabatini DM. An expanding role for mTOR in cancer Trends Mol Med 2005;11:353-361.

15. Mahalingam D, Sankhala K, Mita A, et al. Targeting the mTOR pathway using deforolimus in cancer therapy. Future Oncol 2009;5:291-303.

16. Law BK. Rapamycin: an anti-cancer immunosuppressant? Crit Rev Oncol Hematol 2005;56:47-60.

17. Tee AR, Blenis J. mTOR, translational control and human disease. Semin Cell Dev Biol 2005;16:29-37.

18. Inoki K, Corradetti MN, Guan KL. Dysregulation of the TSC-mTOR pathway in human disease. Nat Genet 2005;37:19-24.

19. Steelman LS, Stadelman KM, Chappell WH, et al. Akt as a therapeutic target in cancer. Expert Opin Ther Targets 2008;12:1139-1165.

20. Jiang BH, Liu LZ. Role of mTOR in anticancer drug resistance: perspective for improved drug treatment. Drug Resist Update 2008;11:63-76.

21. Grant S. Cotargeting survival signaling pathways in cancer. J Clin Invest 2008;118:3003-3006.

22. Harrington LS, Findlay GM, Lamb RF. Restraining P13K: mTOR signalling goes back to the membrane. Trends Biochem Sci 2005; 30:35-42.

23. Le Tourneau $C$, Faivre $S$, Serova $M$, et al. mTORC1 inhibitors: is temsirolimus in renal cancer telling us how they really work? $\mathrm{Br} J$ Cancer 2008;99:1197-1203.

24. Cho D, Signoretti S, Dabora S, et al. Potential histologic and molecular predictors of response to temsirolimus in patients with advanced renal cell carcinoma. Clin Genitourin Cancer 2007;5:379-385.

25. Kong D, Yamori T. Advances in development of phosphatidylinositol 3-kinase inhibitors. Curr Med Chem 2009;16:2839-2854.

26. Liu D, Hou P, Liu Z, et al. Genetic alterations in the phosphoinositide 3-kinase/Akt signaling pathway confer sensitivity of thyroid cacer cells to therapeutic targeting of Akt and mammalian target of rapamycin. Cancer Res 2009;69:7311-7319.

27. Steelman LS, Abrams SL, Whelan J, et al. Contributions of the Raf/MEK/ ERK, P13K/PTEN/Akt/mTOR and Jak/STAT pathways to leukemia. Leukemia 2008;22:686-707.

28. Altomare DA, Testa JR. Pertubations of the AKT signaling pathway in human cancer. Oncogene 2005;24:7455-7464.

29. Lobo GP, Waite KA, Planchon SM, et al. ATP modulates PTEN subcellular localization in multiple cancer cell lines. Hum Mol Genet 2008;17:2877-2885.

30. Tsuruta $\mathrm{H}$, Kishimoto $\mathrm{H}$, Sasaki $\mathrm{T}$, et al. Hyperplasia and carcinomas in PTEN-deficient mice and reduced PTEN protein in human bladder cancer patients. Cancer Res 2006;66:8389-8396.

31. Shen WH, Balajee AS, Wang J, et al. Essential role of nuclear PTEN in maintaining chromosomal integrity. Cell 2007;128:157-170.
32. Ginn-Pease $M E$, Eng C. Increased nuclear phosphatase and tensin homologue deleted on chromosome 10 is associated with G0-G1 in MCF-7 cells. Cancer Res 2003:63:282-286.

33. Chung JH, Eng C. Nuclear-cytoplasmic partitioning of phosphatase and tensin homologue deleted on chromosome 10 (PTEN) differentially regulates the cell cycle and apoptosis. Cancer Res 2005; 65:8096-8100

34. Sun SY, Rosenberg LM, Wang X, et al. Activation of Akt and elF4E survival pathways by rapamycin-mediated mammalian target of rapamycin inhibition. Cancer Res 2005;65:7052-7058.

35. Guo D, Hildebrandt IJ, Prins RM, et al. The AMPK agonist AICAR inhibits the growth of EGFRvlll-expressing glioblastomas by inhibiting lipogenesis. Proc Natl Acad Sci USA 2009;106:12932-12937.

36. Huang J, Manning BD. A complex interplay between Akt, TSC2 and the two mTOR complexes. Biochem Soc Trans 2009;37:217-222.

37. Abdelnour-Berchtold E, Cerantola $Y$, Roulin D, et al. Rapamycinmediated FOXO1 inactivation reduces the anticancer efficacy of rapamycin. Anticancer Res 2010;30:799-804.

38. Cirstea D, Hideshima $\mathrm{T}$, Rodig $\mathrm{S}$, et al. Dual inhibition of akt/ mammalian target of rapamycin pathway by nanoparticle albuminbound-rapamycin and perifosine induces antitumor activity in multiple myeloma. Mol Cancer Ther 2010;9:963-975.

39. Mi R, Ma J, Zhang D, et al. Efficacy of combined inhibition of mTOR and ERK/MAPK pathways in treating a tuberous sclerosis complex cell model. J Genet Genomics 2009;36:355-361.

40. Wang $X$, Hawk $N$, Yue $P$, et al. Overcoming mTOR inhibitioninduced paradoxical activation of survival signaling pathways enhances mTOR inhibitors' anticancer efficacy. Cancer Biol Ther 2008;7: 1952-1958.

41. Sparks CA, Guertin DA. Targeting mTOR: prospects for mTOR complex 2 inhibitors in cancer therapy. Oncogene 2010;29:37333744. Epub: 1-12.

42. Aveyard JS, Skilleter A, Habuchi T, et al. Somatic mutation of PTEN in bladder carcinoma. Br J Cancer 1999;80:904-908.

43. Theodorescu D, Laderoute KR, Gulding KM. Epidermal growth factor receptor-regulated human bladder cancer motility is in part a phosphatidylinositol 3-kinase-mediated process. Cell Growth Differ 1998;9:919-928.

44. Gildea JJ, Herlevsen M, Harding MA, et al. PTEN can inhibit in vitro organotypic and in vivo orthotopic invasion of human bladder cancer cells even in the absence of its lipid phosphatase activity. Oncogene 2004;23:6788-6797.

45. Gildea JJ, Harding MA, Seraj MJ, et al. The role of Ral A in epidermal growth factor receptor-regulated cell motility. Cancer Res 2002; 62:982-985.

46. Kanda S, Kanetake H, Miyata Y. Loss of PTEN function may account for reduced proliferation pathway sensitivity to LY294002 in human prostate and bladder cancer cells. J Cancer Res Clin Oncol 2009; 135:303-311.

47. Saal LH, Johansson P, Holm K, et al. Poor prognosis in carcinoma is associated with a gene expression signature of aberrant PTEN tumor suppressor pathway activity. Proc Natl Acad Sci USA 2007; 104:7564-7569.

48. Knowles MA, Platt FM, Ross RL, et al. Phosphatidylinositol 3-kinase (PI3K) pathway activation in bladder cancer. Cancer Metastasis Rev 2009;28:305-316.

49. Platt FM, Hurst CD, Tayler CF, et al. Spectrum of phosphatidylinositol 3kinase pathway gene alterations in bladder cancer. Clin Cancer Res 2009;15:6008-6017.

50. Ikenoue T, Kanai F, Hikiba Y, et al. Functional analysis of PIK3CA gene mutations in human colorectal cancer. Cancer Res 2005:65:4562-4567.

51. Zhao L, Vogt PK. Hot-spot mutations in p110alpha of phosphatidylinositol 3-kinase (PI3K): diffferential interactions with the regulatory subunit p85 and with RAS. Cell Cycle; 9:596-600.

52. Qian CN, Furge KA, Knol J, et al. Activation of the PI3K/Akt pathway induces urothelial carcinoma of the renal pelvis: idenitifcation in human tumors and confirmation in animal models. Cancer Res 2009;69:8256-8264.

53. Lopez-Knowles E, Hernandez S, Malats N, et al. PIK3CA mutations are an early genetic alteration associated with FGFR3 mutations in superficial papillary bladder tumors. Cancer Res 2006;66:7401-7404.

54. Zhao L, Vogt PK. Class I PI3K in oncogenic cellular transformation. Oncogene 2008;27:5486-5496. 
55. Huang $\mathrm{CH}$, Mandelker D, Gabelli SB, et al. Insights into the oncogenic effects of PIK3CA mutations from the structure of p110alpha/ p85alpha. Cell Cycle 2008;7:1151-1156.

56. Philp AJ, Campbell IG, Leet C, et al. The phosphatidylinositol 3'-kinase p85alpha gene is an oncogene in human ovarian and colon tumors. Cancer Res 2001;61:7426-7429.

57. Jiang $\mathrm{BH}$, Liu LZ. PI3K/PTEN signaling in tumorigenesis and angiogenesis. Biochem Biophys Acta 2008;1784:150-158.

58. Matheny Jr RW, Adamo ML. Current perspectives on Akt Akt-ivation and Akt-ions. Exp Biol Med 2009;234:1264-1270.

59. Wu X, Obata $T$, Khan $Q$, et al. The phosphatidylinositol-3 kinase pathway regulates bladder cancer cell invasion. $\mathrm{Br} J$ Urol Int 2004;93:143-150.

60. Harris LD, De La Cerda J, Tuziak T, et al. Analysis of the expression of biomarkers in urinary bladder cancer using a tissue microarray. Mol Carcinog 2008;47:678-685.

61. Oka N, Tanimoto S, Taue R, et al. Role of phosphatidylinositol-3 kinase/ Akt pathway in bladder cancer cell apoptosis induced by tumor necrosis factor-related apoptosis-inducing ligand. Cancer Sci 2006; 97:1093-1098.

62. Szanto A, Bognar Z, Szigeti A, et al. Criticlal role of bad phosphorylation by Akt in cytostatic resistance of human bladder cancer. Anticancer Res 2009;29:159-164.

63. Sun L, Zhao H, Xu Z, et al. Phosphatidylinositol 3-kinase/protein kinase $B$ pathway stabilizes DNA methyltransferase I protein and maintains DNA methylation. Cell Signal 2007;19:2255-2263.

64. Askham JM, Platt F, Chambers PA, et al. AKT1 mutations in bladder cancer: identification of a novel oncogenic mutation that can cooperate with E17K. Oncogene 2010;29:150-155.

65. Cairns $P$, Shaw ME, Knowles MA. Initiation of bladder cancer may involve deletion of a tumour-suppressor gene on chromosome 9. Oncogene 1993;8:1083-1085.

66. Pymar LS, Platt FM, Askham JM, et al. Bladder tumour-derived somatic TSC1 missense mutations cause loss of function via distinct mechanisms. Hum Mol Genet 2008;17:2006-2017.

67. Rosner M, Fuchs C, Siegel N, et al. New insights into the role of tuberous sclerosis gene in leukemia. Leuk Res 2009;33:883-885.

68. Hashimoto I, Koizumi K, Tatematsu M, et al. Blocking on the CXCR4/ mTOR signalling pathway induces the anti-metastatic properties and autophagic cell death in peritoneal disseminated gastric cancer cells. Eur J Cancer 2008;44:1022-1029.

69. Lamouille S, Derynck R. Cell size and invasion in TGF-beta-induced epithelial to mesenchymal transition is regulated by activation of the mTOR pathway. J Cell Biol 2007;178:437-451.

70. Faried LS, Faried A, Kanuma T, et al. Predictive and prognostic role of activated mammalian target of rapamycin in cervical cancer treated with cisplatin-based neoadjuvant chemotherapy. Oncol Rep 2006; 16:57-63.
71. Hansel DE, Platt $\mathrm{E}$, Orloff $\mathrm{M}$, et al. Mammalian Target of Rapamycin (mTOR) regulates cellular proliferation and tumor growth in urothelial carcinoma. Am J Pathol; 176:3062-3072.

72. Seager CM, Puzio-Kuter AM, Patel $\mathrm{T}$, et al. Intravesical delivery of rapamycin suppresses tumorigenesis in a mouse model of progressive bladder cancer. Cancer Prev Res 2009;2:1008-1014.

73. Sabatini DM. mTOR and cancer: insights into a complex relationship. Nat Rev 2006;6:729-734.

74. Bansal N, Yendiuri V, Wenham RM. The molecular biology of endometrial cancers and the implications for pathogenesis, classification, and targeted therapy. Cancer Control 2009;16:8-13.

75. Hudes G, Carducci M, Tomczak $P$, et al. A phase 3, randomized, 3-arm study of temsirolimus (TEMSR) or interferon-alpha (IFN) or the combination of TEMSR + IFN in the treatment of first-line, poor-risk patients with advanced renal cell carcinoma (adv RCC). J Clin Oncol 2006;24(18 suppl):2s.

76. Atkins $M B$, Hidalgo $M$, Stadler WM, et al. Randomized phase II study of multiple dose levels of $\mathrm{CCl}-779$, a novel mammalian target of rapamycin kinase inhibitor, in patients with advanced refractory renal cell carcinoma. J Clin Oncol 2004;22:909-918.

77. Qin J, Xie LP, Zheng XY, et al. A component of green tea, (-)epigallocatechin-3-gallate, promotes apoptosis in T24 human bladder cancer cells via modulation of the PI3K/Akt pathway and $\mathrm{BCl}-2$ family proteins. Biochem Biophys Res Commun 2007; 354:852-857.

78. Rieger-Christ KM, Hanley R, Lodowsky C, et al. The green tea compound, (-)-epigallocatechin-3-gallate downregulates $\mathrm{N}$-cadherin and suppresses migration of bladder carcinoma cells. J Cell Biochem 2007;102:377-388.

79. Engleman JA. Targeting PI3K signalling in cancer: opportunities, challenges and limitations. Nature 2009;9:550-562.

80. Wu P, Tao L, Hu Y. PI3K inhibitors for cancer therapy: what has been achieved so far? Curr Med Chem 2009;16:916-930.

81. Berrie CP. Phosphoinositide 3-kinase inhibition in cancer treatment. Expert Opin Investig Drugs 2001;10:1085-1098.

82. Hu L, Zaloudek C, Mills GB, et al. In vivo and in vitro ovarian carcinoma growth inhibition by a phosphatidylinositol 3-kinase inhibito (LY294002). Clin Cancer Res 2000;6:880-886.

83. Gupta AK, Cerniglia GJ, Mick R, et al. Radiation sensitization of human cancer cells in vivo by inhibiting the activity of PI3K using LY294002. Int J Radiat Oncol Biol Phys 2003;56:846-853.

84. Kong D, Yamori T. Phosphatidylinositol 3-kinase inhibitors: promising drug. Cancer Sci 2008;99:1734-1740.

85. Liu $\mathrm{P}$, Cheng $\mathrm{H}$, Roberts $\mathrm{T}$, et al. Targeting the phosphoinositide 3-kinase pathway in cancer. Nat Rev Drug Discov 2009;8:627-644.

86. Vink SR, Schellens $\mathrm{JH}$, Beinen $\mathrm{JH}$, et al. Phase I and pharmacokinetic study of combined treatment with perifosine and radiation in patients with advanced solid tumors. Radiother Oncol 2006;80:207-213. 